\title{
On the relationship among F-transform, fuzzy rough set and fuzzy topology
}

\author{
Irina Perfilieva ${ }^{1}$ Anand Pratap Singh ${ }^{2}$ S.P. Tiwari ${ }^{2}$ \\ ${ }^{1}$ University of Ostrava, Institute for Research and Applications of Fuzzy Modeling \\ NSC IT4Innovations, 30. dubna 22, 70103 Ostrava 1, Czech Republic \\ ${ }^{2}$ Dept. of Applied Mathematics, Indian School of Mines, Dhanbad-826004, India
}

\begin{abstract}
The objective of this work is to associate the concepts of fuzzy rough sets and fuzzy topologies with fuzzy transform. It is shown here that the fuzzy transform can be viewed as fuzzy approximation operators studied in the operator-oriented view of fuzzy rough set theory and the use of fuzzy rough set results reduces efforts in proving fuzzy transform theoretic results. We present a glimpse that fuzzy topological results can also be used in similar fashion for fuzzy transform theoretical results.
\end{abstract}

Keywords: F-transform, Fuzzy partition, Residuated lattices, Fuzzy rough sets.

\section{Introduction}

Fuzzy transform ( $F$-transform), firstly proposed by Perfilieva in [10] has now been significantly developed and opened a new page in the theory of semilinear spaces. The main idea of the $F$-transform is to factorize (or fuzzify) precise values of independent variables by a closeness relation, and precise values of dependent variables are averaged to an approximate value. It is shown in [10] that this transform encompassed both classical transform as well as approximation methods based on fuzzy IFTHEN rules studied in fuzzy modeling. The theory of $F$-transform was further elaborated and extended from real valued to lattice-valued functions (cf., [10, 12]) and from fuzzy sets to parametrized fuzzy sets (cf., [17]). The theory of $F$-transform is successfully used in signal and image processing [7], compression [11], denoising [9], numerical solutions of partial differential equations [18], data analysis [13], and neural network approaches [19].

In recent years, another theory that has drawn the attention of researchers is the rough set theory, proposed by Pawlak [8]. This theory has been developed significantly due to its importance for the study of intelligent systems with insufficient and incomplete information. In rough sets introduced by Pawlak, the key role is played by equivalence relations. In literature $[5,15,22]$, several generalizations of rough sets have been made by replacing the equivalence relation by an arbitrary relation. After Dubois and Prade [3] introduced a fuzzy rough set, which is a generalization of a rough set, the relationship between fuzzy rough sets and fuzzy topological spaces were studied $[1,14,20,21]$.

Both concepts ( $F$-transform and fuzzy rough set) gained popularity. The first one is based on a fuzzy partition of a universe, and the second one is based on a fuzzy relation. A close look to both theories leads to a conclusion that an interrelationship between them can be established so that the concept of the fuzzy rough set theory may play an important role in the development of the theory of $F$ transforms. To establish such relationship is the main topic of this paper. Specifically, we show that the $F$-transforms introduced in [10] can be viewed as approximation operators studied in the operatororiented view of fuzzy rough set theory. We show that the use of fuzzy rough set theory reduces in a large number of cases our effort in proving $F$ transform theoretic results. Furthermore, as the concept of fuzzy topology can naturally be associated with the theory of fuzzy rough sets, there is a possibility of a good trade-off between the theory of fuzzy topology and the theory of $F$-transform.

\section{Preliminaries}

In this section, we recall some concepts related to residuated lattices, fuzzy rough sets and fuzzy topology, which we will need in the subsequent sections. We begin with the definition taken from [2].

Definition 2.1 $A$ residuated lattice is an algebra $(L, \wedge, \vee, *, \rightarrow, 0,1)$ such that

(i) $(L, \wedge, \vee, 0,1)$ is a bounded lattice with the least element 0 and the greatest element 1 ;

(ii) $(L, *, 1)$ is a commutative monoid;

(iii) $\forall a, b, c \in L$

$$
a * b \leq c \text { iff } a \leq b \rightarrow c,
$$

i.e., $(\rightarrow, *)$ is an adjoint pair on $L$.

A residuated lattice $(L, \wedge, \vee, *, \rightarrow, 0,1)$ is complete if it is complete as a lattice. For $a \in L$, the operations of negation and biresiduation are defined by

$$
\begin{aligned}
\neg a & =a \rightarrow 0, \\
a \leftrightarrow b & =(a \rightarrow b) \wedge(b \rightarrow a) .
\end{aligned}
$$


Proposition 2.1 [2] Let $(L, \wedge, \vee, *, \rightarrow, 0,1)$ be a residuated lattice. Then for all $a, b \in L$,

(i) $a \leq(b \rightarrow a * b)$;

(ii) $a *(a \rightarrow b) \leq b$;

(iii) $a *\left(\vee_{i \in I} b_{i}\right)=\vee_{i \in I}\left(a * b_{i}\right)$;

(iv) $a \rightarrow 1=1,1 \rightarrow a=a$;

(v) $a \rightarrow \bigwedge_{i \in I} b_{i}=\bigwedge_{i \in I}\left(a \rightarrow b_{i}\right), \bigvee_{i \in I} a_{i} \rightarrow b=$ $\bigwedge\left(a_{i} \rightarrow b\right)$.

Throughout this paper, we work with a complete residuated lattice $L=([0,1], \wedge, \vee, *, \rightarrow, 0,1)$.

The fuzzy sets considered in this paper take membership degrees from $L$. For a nonempty set $X, L^{X}$ denotes the collection of all fuzzy subsets of $X$. For all $a \in L, \mathbf{a}(x)=a$ denotes the constant fuzzy set. The $\operatorname{core}(A)$ is a set of all elements $x \in X$ such that $A(x)=1$. A fuzzy set $A \in L^{X}$ is normal if $\operatorname{core}(A) \neq \emptyset$.

Definition $2.2[20]$ Let $X$ be a nonempty set. Then for $A, B \in L^{X}$ and $x \in X$. Then

(i) $(A * B)(x)=A(x) * B(x)$;

(ii) $(A \rightarrow B)(x)=A(x) \rightarrow B(x)$;

(iii) $(\neg A)(x)=A(x) \rightarrow 0$.

Definition 2.3 [4] Let $X$ be a nonempty set. A fuzzy relation $R$ on $X$ is a fuzzy subset of $X \times X$. A fuzzy relation $R$ is called

(i) reflexive if $R(x, x)=1, \forall x \in X$;

(ii) transitive if $R(x, y) * R(y, z) \leq$ $R(x, z), \forall x, y, z \in X$. A reflexive and transitive fuzzy relation $R$ is called a fuzzy preorder.

Now we recall the following concepts associated with fuzzy rough sets.

Definition 2.4 [21] A pair $(X, R)$ is called a fuzzy approximation space if $X$ is a nonempty set and $R$ is a fuzzy relation on $X$.

Definition 2.5 [21] Let $(X, R)$ be a fuzzy approximation space. Then the two functions $\underline{R}, \bar{R}$ : $L^{X} \longrightarrow L^{X}$, defined, $\forall A \in L^{X}, \forall x \in X$,

$$
\begin{aligned}
& \underline{R}(A)(x)=\bigwedge_{y \in X}(R(x, y) \rightarrow A(y)) ; \\
& \bar{R}(A)(x)=\bigvee_{y \in X}(R(x, y) * A(y)) .
\end{aligned}
$$

are called the lower fuzzy approximation operator and the upper fuzzy approximation operator, respectively.

Proposition 2.2 [20] Let $(X, R)$ be a fuzzy approximation space, $a \in L, A, B \in L^{X}$ and $\left\{A_{i}\right\}_{i \in I} \subseteq$ $L^{X}$. Then:

(i) if $A \subseteq B$ then $\underline{R}(A) \subseteq \underline{R}(B)$ and $\bar{R}(A) \subseteq$ $\bar{R}(B)$;

(ii) $\bar{R}\left(\bigcup_{i \in I} A_{i}\right)=\bigcup_{i \in I} \bar{R}\left(A_{i}\right), \underline{R}\left(\bigcap_{i \in I} A_{i}\right)=$ $\bigcap_{i \in I} \underline{R}\left(A_{i}\right)$; (iii) $\bar{R}(a * A)=a * \bar{R}(A), \underline{R}(a \rightarrow A)=a \rightarrow \underline{R}(A)$.

(iv) $\bar{R}(\mathbf{a})=\mathbf{a}, \underline{R}(\mathbf{a})=\mathbf{a}$.

The fuzzy topological concepts we use here are fairly standard and can be found in the literature [6]. For completeness, we recall the following key notions; for other notions, readers are referred to Lowen [6].

Proposition 2.3 [16] Let $(X, R)$ be a fuzzy approximation space, $A \in L^{X}$. Then,

(i) $R$ is reflexive $\Longleftrightarrow \underline{R}(A) \subseteq A \Longleftrightarrow A \subseteq \bar{R}(A)$;

(ii) $R$ is Euclidean $\Longleftrightarrow \bar{R}(A) \subseteq \underline{R}(\bar{R}(A)) \Longleftrightarrow$ $\bar{R}(\underline{R}(A)) \subseteq \underline{R}(A)$.

Definition $2.6[6] A$ fuzzy topology $\tau$ on a nonempty set $X$ is a family of fuzzy sets in $X$ which is closed under arbitrary suprema and finite infima and contains all constant fuzzy sets. The fuzzy sets in $\tau$ are called open, and their complements, closed.

Definition $2.7[6] \quad A \quad$ Kuratowski fuzzy closure operator on $X$ is a map $c: L^{X} \longrightarrow L^{X}$ satisfying $\forall a \in L, A, B \in L^{X}$,

(i) $c(\mathbf{a})=\mathbf{a}$;

(ii) $A \leq c(A)$;

(iii) $c(A \vee B)=c(A) \vee c(B)$;

(iv) $c(c(A))=c(A)$.

Remark 2.1 Every Kuratowski fuzzy closure operator $c$ on a set $X$ gives rise to a fuzzy topology on $X$ in which a fuzzy set $\mu$ is closed iff $c(\mu)=\mu$.

Definition 2.8 [6] A Kuratowski fuzzy interior operator on $X$ is a map $i: L^{X} \longrightarrow L^{X}$ satisfying $\forall a \in L, A, B \in L^{X}$,

(i) $i(\mathbf{a})=\mathbf{a}$;

(ii) $i(A) \leq A$;

(iii) $i(A \wedge B)=i(A) \wedge i(B)$;

(iv) $i(i(A))=i(A)$.

Remark 2.2 Every Kuratowski fuzzy interior operator $i$ on a set $X$ gives rise to a fuzzy topology on $X$ in which a fuzzy set $\mu$ is open iff $i(\mu)=\mu$.

We close this section by recalling the following two theorems from [20].

Theorem 2.1 Let $(X, R)$ be fuzzy approximation space and $c: L^{X} \longrightarrow L^{X}$ be a Kuratowski fuzzy closure operator. Then there exists a fuzzy preorder $R$ on $X$ such that $\bar{R}(A)=c(A)$, for all $A \in L^{X}$ iff $c: L^{X} \longrightarrow L^{X}$ satisfies the following conditions:

(i) $c\left(\bigcup_{j \in J} A_{j}\right)=\bigcup_{j \in J} c\left(A_{j}\right) ; A_{j} \in L^{X}, j \in J$,

(ii) $c(A * a)=c(A) * a, a \in L$.

Theorem 2.2 Let $(X, R)$ be fuzzy approximation space and $i: L^{X} \longrightarrow L^{X}$ be Kuratowski fuzzy interior operator. Then there exists fuzzy preorder $R$ on $X$ such that $\underline{R}(A)=i(A)$, for all $A \in L^{X}$ iff $i: L^{X} \longrightarrow L^{X}$ satisfies the following conditions:

(i) $i\left(\bigcap_{j \in J} A_{j}\right)=\bigcap_{j \in J} i\left(A_{j}\right) ; A_{j} \in L^{X}, j \in J$,

(ii) $i(a \rightarrow A)=a \rightarrow i(A), a \in L$. 


\section{F-transform, fuzzy approximation operators and fuzzy topology}

In this section, we show that the direct $F^{\uparrow}\left(F^{\downarrow}\right)$ transform can be viewed as an upper(lower) fuzzy approximation operator in the context of fuzzy rough set theory. Throughout, we will work with general universal set $X$ and residuated lattice $L$.

Definition 3.1 Let $n \geq 2$. A collection of normal fuzzy sets $A_{1}, \ldots, A_{k}, \ldots$, defined on $X$, is a fuzzy partition of $X$, if the corresponding collection of ordinary sets core $\left(A_{1}\right), \ldots$, core $\left(A_{k}\right), \ldots$ is a partition of $X$.

A fuzzy partition $A_{1}, \ldots, A_{k}, \ldots$ of $X$ can be represented by the following reflexive fuzzy relation

$$
R_{A_{1}, \ldots, A_{k}, \ldots}(x, y)=A_{k}(y) \text {, if } x \in \operatorname{core}\left(A_{k}\right) .
$$

For each particular $k=1, \ldots, n, \ldots$, we define fuzzy relation $R_{k}$ on $X$ :

$$
R_{k}(x, y)= \begin{cases}A_{k}(y), & \text { if } x \in \operatorname{core}\left(A_{k}\right) \\ 1, & \text { if } x=y \\ 0, & \text { otherwise }\end{cases}
$$

It can be easily seen that fuzzy relation $R_{k}$ is reflexive. Let us prove that $R_{k}$ is a fuzzy preorder.

Proposition 3.1 Let fuzzy relation $R_{k}$ be given in (2). Then it is a fuzzy preorder on $X$.

\section{Proof:}

We need to verify that $R_{k}$ is transitive. Let us choose $x, y, z \in X$ and show that $R_{k}(x, y) *$ $R_{k}(y, z) \leq R_{k}(x, z)$. We will consider four possible cases:

1. If $x, y \in \operatorname{core}\left(A_{k}\right)$, then

$$
\begin{aligned}
R_{k}(x, y) * R_{k}(y, z)=A_{k}(y) * A_{k}(z) & \leq A_{k}(z) \\
& =R_{k}(x, z)
\end{aligned}
$$

2. If $x \in \operatorname{core}\left(A_{k}\right)$ and $y \notin \operatorname{core}\left(A_{k}\right)$, then

(a) if $y=z$, then

$$
\begin{aligned}
R_{k}(x, y) * R_{k}(y, z)=A_{k}(y) * 1 & =A_{k}(z) \\
& =R_{k}(x, z)
\end{aligned}
$$

(b) If $y \neq z$, then

$$
R_{k}(x, y) * R_{k}(y, z)=A_{k}(y) * 0=0 \leq R_{k}(x, z) ;
$$

3. If $x \notin \operatorname{core}\left(A_{k}\right)$ and $y \in \operatorname{core}\left(A_{k}\right)$, then the proof is similar to the above given;

4. If $x \notin \operatorname{core}\left(A_{k}\right)$ and $y \notin \operatorname{core}\left(A_{k}\right)$, then

(a) if $x=y$, then

$$
\begin{array}{rl}
R_{k}(x, y) * R_{k}(y, z)=1 & * R_{k}(y, z)= \\
& R_{k}(y, z)=R_{k}(x, z)
\end{array}
$$

(b) if $x \neq y$, then

$$
R_{k}(x, y) * R_{k}(y, z)=0 * R_{k}(y, z)=0 \leq R_{k}(x, z) .
$$

It is easy to see that for any fuzzy partition $A_{1}, \ldots, A_{k}, \ldots$ of $X$, the representing relation $R_{A_{1}, \ldots, A_{k}, \ldots}$ can be decomposed into the union of fuzzy preorder relations $R_{k}$ :

$$
R_{A_{1}, \ldots, A_{k}, \ldots}=\bigcup_{k} R_{k}
$$

\subsection{Direct $F^{\uparrow}\left(F^{\downarrow}\right)$-transform}

In this subsection, we will generalize the notion of the direct $F^{\uparrow}\left(F^{\downarrow}\right)$-transform to the case where a transformed function is defined on an arbitrary set $X$ and takes values from the residuated lattice support $L$. We will show that the generalized $F^{\uparrow}\left(F^{\downarrow}\right)$-transform can be viewed as an upper (lower) fuzzy approximation operator and hence induces fuzzy topology on the respective universe. We also show that the results regarding the direct $F^{\uparrow}\left(F^{\downarrow}\right)$-transform in [10] can be obtained as consequences of well-known concepts from fuzzy rough set theory and fuzzy topology. We begin with the following new concept of the direct upper $F^{\uparrow}$ transform.

Definition 3.2 [10] Let $f$ be an L-valued function on $X$ (in other words, a fuzzy set) and $A_{1}, \ldots, A_{k}, \ldots$ be a fuzzy partition of $X$. The (upper) $F^{\uparrow}$-transform of $f$ w.r.t. $A_{1}, \ldots, A_{k}, \ldots$ is a sequence of lattice elements $\left(F_{1}^{\uparrow}, \ldots, F_{k}^{\uparrow}, \ldots\right)$, where

$$
F_{k}^{\uparrow}=\bigvee_{x \in X}\left(A_{k}(x) * f(x)\right), k \geq 1
$$

We denote $F^{\uparrow}[f]=\left(F_{1}^{\uparrow}, \ldots, F_{k}^{\uparrow}, \ldots\right)$ the $F^{\uparrow}$ transform of $f$ and $F_{k}^{\uparrow}[f]$ (or simply $F_{k}^{\uparrow}$, if $f$ is clear from the context) its $k$-th component.

Let $R_{A_{1}, \ldots, A_{k}, \ldots}$ be a representing relation of fuzzy partition $A_{1}, \ldots, A_{k}, \ldots$ of $X$. Then in fuzzy approximation space $\left(X, R_{A_{1}, \ldots, A_{k}, \ldots}\right)$, the following holds:

$$
\bar{R}_{A_{1}, \ldots, A_{k}, \ldots}(f)(x)=F_{k}^{\uparrow}[f] \text {, if } x \in \operatorname{core}\left(A_{k}\right), k \geq 1 \text {. }
$$

consequence of (5), we have the following

Proposition 3.2 Let $\left.X\right|_{A_{1}, \ldots, A_{k}, \ldots}=$ $\left\{x_{1}, \ldots, x_{k}, \ldots\right\}$, where $x_{k} \in \operatorname{core}\left(A_{k}\right), k \geq 1$. Then the $F^{\uparrow}$-transform of $f F^{\uparrow}[f]$ is a reduction of the upper approximation $\bar{R}_{A_{1}, \ldots, A_{k}, \ldots}(f)$ on $\left.X\right|_{A_{1}, \ldots, A_{k}, \ldots}$.

Below, we analyze properties of particular components $F_{k}^{\uparrow}, k \geq 1$.

Proposition 3.3 Let $R_{k}, k=1, \ldots, n, \ldots$ be a constituent fuzzy preorder relation (2) in the decomposition (3). Then 
1. in fuzzy approximation space $\left(X, R_{k}\right), k \geq 1$, the upper approximation of an L-valued function $f$ is equal to

$$
\bar{R}_{k}(f)(x)= \begin{cases}F_{k}^{\uparrow}[f], & x \in \operatorname{core}\left(A_{k}\right), \\ f(x), & \text { otherwise }\end{cases}
$$

2. the $k$-th upper F-transform component $F_{k}^{\uparrow}$ of $f$ is a reduction of the upper approximation $\bar{R}_{k}(f)$ on $\operatorname{core}\left(A_{k}\right)$, so that

$$
F_{k}^{\uparrow}[f]=\left.\bar{R}_{k}(f)\right|_{\text {core }\left(A_{k}\right)}=\bar{R}_{k}(f)\left(x_{k}\right),
$$

where $x_{k} \in \operatorname{core}\left(A_{k}\right)$.

\section{Proof:}

1. If $x \in \operatorname{core}\left(A_{k}\right)$, then by (2),

$$
\begin{aligned}
\bar{R}_{k}(f)(x)=\bigvee_{y \in X}\left(R_{k}(x, y) * f(y)\right) & = \\
\bigvee_{y \in X}\left(A_{k}(y) * f(y)\right) & =F_{k}^{\uparrow}[f]
\end{aligned}
$$

If on the other side, $x \notin \operatorname{core}\left(A_{k}\right)$, then by (2),

$$
\bar{R}_{k}(f)(x)=\bigvee_{y \in X}\left(R_{k}(x, y) * f(y)\right)=f(x) .
$$

Thus, (6) is confirmed.

2. Let $x_{k} \in \operatorname{core}\left(A_{k}\right)$. Then by (6),

$$
F_{k}^{\uparrow}[f]=\bar{R}_{k}(f)\left(x_{k}\right)=\left.\bar{R}_{k}(f)\right|_{\operatorname{core}\left(A_{k}\right)} .
$$

On the basis of the Proposition 3.3, upper approximation $\bar{R}_{k}(f)$ is called the $k$-th upper F-transform approximation of $f$.

Being a reduction of the upper approximation operator $\bar{R}_{k}$, the $k$-th component $F_{k}^{\uparrow}$ fulfills all its properties introduced above. In particular, we have

Proposition 3.4 Let $a, \alpha, \beta \in L, f, g$ be L-valued functions on $X$ and $A_{1}, \ldots, A_{k}, \ldots$ be a fuzzy partition of $X$. Then for all $k \geq 1$,

1. $F_{k}^{\uparrow}[\mathbf{a}]=\mathbf{a}$,

2. $F_{k}^{\uparrow}[\alpha * f \vee \beta * g]=\alpha * F_{k}^{\uparrow}[f] \vee \beta * F_{k}^{\uparrow}[g]$,

3. $F_{k}^{\uparrow}[f] \leq F_{k}^{\uparrow}[g]$, if $f \leq g$,

4. $f\left(x_{k}\right) \leq F_{k}^{\uparrow}[f]$, if $x_{k} \in \operatorname{core}\left(A_{k}\right)$.

Proof: This follows from Proposition 2.2.

The following proposition is a consequence of Theorem 2.1, so that it connects a $k$-th upper Ftransform approximation with a closure operator in the corresponding fuzzy topology.

Proposition 3.5 Let $R_{A_{1}, \ldots, A_{k}, \ldots}$ be a representing relation of fuzzy partition $A_{1}, \ldots, A_{k}, \ldots$ of $X$ and $R_{k}, k=1, \ldots, n, \ldots$ be a constituent fuzzy preorder relation (2) in the decomposition (3). Let us define the operator $c_{k}: L^{X} \rightarrow L^{X}$ as the $k$-th upper $F$ transform approximation, i.e. for any $f \in L^{X}$,

$$
c_{k}(f)=\bar{R}_{k}(f) .
$$

Then $c_{k}$ is a Kuratowski fuzzy closure operator in the topology that is determined by fuzzy preorder relation $R_{k}$.

Proof: By Proposition 3.4, operator $c_{k}$ fulfills all properties, listed in Definition 2.7, and the two additional properties, listed in Theorem 2.1. Therefore, $c_{k}$ is a Kuratowski fuzzy closure operator and there exists a fuzzy preorder $R$ on $X$ such that for all $A \in L^{X}, \bar{R}(A)=c_{k}(A)$. It is easy to see that $R_{k}$ can be chosen for $R$.

Now, we show that the similar results can be also obtained for the direct lower $F^{\downarrow}$-transform.

Definition 3.3 [10] Let $f$ be an L-valued function on $X$ (in other words, a fuzzy set) and $A_{1}, \ldots, A_{k}, \ldots$ be a fuzzy partition of $X$. The (lower) $F^{\downarrow}$-transform of $f$ w.r.t. $A_{1}, \ldots, A_{k}, \ldots$ is a sequence of lattice elements $\left(F_{1}^{\downarrow}, \ldots, F_{k}^{\downarrow}, \ldots\right)$, where

$$
F_{k}^{\downarrow}=\bigwedge_{x \in X}\left(A_{k}(x) \rightarrow f(x)\right), k \geq 1 .
$$

We denote $F^{\downarrow}[f]=\left(F_{1}^{\downarrow}, \ldots, F_{k}^{\downarrow}, \ldots\right)$ and $F_{k}^{\downarrow}[f]$ the $F^{\downarrow}$-transform of $f$ and its $k$-th component, respectively.

Let $R_{A_{1}, \ldots, A_{k}, \ldots}$ be a representing relation of fuzzy partition $A_{1}, \ldots, A_{k}, \ldots$ of $X$. Then in fuzzy approximation space $\left(X, R_{A_{1}, \ldots, A_{k}, \ldots}\right)$, the following holds:

$$
\underline{R}_{A_{1}, \ldots, A_{k}, \ldots}(f)(x)=F_{k}^{\downarrow}[f] \text {, if } x \in \operatorname{core}\left(A_{k}\right), k \geq 1 .
$$

As a consequence of (9), we have the following

Proposition 3.6 Let $\left.X\right|_{A_{1}, \ldots, A_{k}, \ldots}=$ $\left\{x_{1}, \ldots, x_{k}, \ldots\right\}$, where $x_{k} \in \operatorname{core}\left(A_{k}\right), k \geq 1$. Then the $F^{\downarrow}$-transform of $f F^{\downarrow}[f]$ is a reduction of the lower approximation $\underline{R}_{A_{1}, \ldots, A_{k}, \ldots}(f)$ on $\left.X\right|_{A_{1}, \ldots, A_{k}, \ldots}$.

Below, we analyze properties of particular components $F_{k}^{\downarrow}, k \geq 1$.

Proposition 3.7 Let $R_{k}, k=1, \ldots, n, \ldots$ be a constituent fuzzy preorder relation (2) in the decomposition (3). Then

1. in fuzzy approximation space $\left(X, R_{k}\right), k \geq 1$, the lower approximation of an $L$-valued function $f$ is equal to

$$
\underline{R}_{k}(f)(x)= \begin{cases}F_{k}^{\downarrow}[f], & x \in \operatorname{core}\left(A_{k}\right), \\ f(x), & \text { otherwise, }\end{cases}
$$


2. the $k$-th lower F-transform component $F_{k}^{\downarrow}[f]$ of $f$ is a reduction of the lower approximation $\underline{R}_{k}(f)$ on $\operatorname{core}\left(A_{k}\right)$, so that

$$
F_{k}^{\downarrow}[f]=\left.\underline{R}_{k}(f)\right|_{\operatorname{core}\left(A_{k}\right)}=\underline{R}_{k}(f)\left(x_{k}\right),
$$

where $x_{k} \in \operatorname{core}\left(A_{k}\right)$.

\section{Proof:}

1. If $x \in \operatorname{core}\left(A_{k}\right)$, then by $(2)$,

$$
\begin{aligned}
\underline{R}_{k}(f)(x)= & \bigwedge_{y \in X}\left(R_{k}(x, y) \rightarrow f(y)\right)= \\
& \bigwedge_{y \in X}\left(A_{k}(y) \rightarrow f(y)\right)=F_{k}^{\downarrow}[f] .
\end{aligned}
$$

If on the other hand, $x \notin \operatorname{core}\left(A_{k}\right)$, then by (2),

$$
\underline{R}_{k}(f)(x)=\bigwedge_{y \in X}\left(R_{k}(x, y) \rightarrow f(y)\right)=f(x) .
$$

Thus, (10) is confirmed.

2. Let $x_{k} \in \operatorname{core}\left(A_{k}\right)$. Then by (10),

$$
F_{k}^{\downarrow}[f]=\underline{R}_{k}(f)\left(x_{k}\right)=\left.\underline{R}_{k}(f)\right|_{\operatorname{core}\left(A_{k}\right)} .
$$

On the basis of the Proposition 3.7, lower approximation $\underline{R}_{k}(f)$ is called the $k$-th lower F-transform approximation of $f$.

Being a reduction of the lower approximation operator $\underline{R}_{k}$, the $k$-th component $F_{k}^{\downarrow}$ fulfills all its properties introduced above. In particular, we have the following.

Proposition 3.8 Let $\alpha, \beta \in L, f, g$ be L-valued functions on $X$ and $A_{1}, \ldots, A_{k}, \ldots$ be a fuzzy partition of $X$. Then for all $k \geq 1$,

1. $F_{k}^{\downarrow}[\mathbf{a}]=\mathbf{a}$,

2. $F_{k}^{\downarrow}[(\alpha \rightarrow f) \wedge(\beta \rightarrow g)]=$

3. $F_{k}^{\downarrow}[f] \leq F_{k}^{\downarrow}[g]$, if $f \leq g$,

$$
\left(\alpha \rightarrow F_{k}^{\downarrow}[f]\right) \wedge\left(\beta \rightarrow F_{k}^{\downarrow}[g]\right),
$$

4. $f\left(x_{k}\right) \geq F_{k}^{\downarrow}[f]$, if $x_{k} \in \operatorname{core}\left(A_{k}\right)$.

Proof: This follows from Proposition 2.2.

Similarly and dually to the above, the following proposition is a consequence of Theorem 2.2, so that it connects a $k$-th lower $\mathrm{F}$-transform approximation with an interior operator in the corresponding fuzzy topology.

Proposition 3.9 Let $R_{A_{1}, \ldots, A_{k}, \ldots}$ be a representing relation of fuzzy partition $A_{1}, \ldots, A_{k}, \ldots$ of $X$ and $R_{k}, k=1, \ldots, n, \ldots$ be a constituent fuzzy preorder relation (2) in the decomposition (3). Let us define the operator $d_{k}: L^{X} \rightarrow L^{X}$ as the $k$-th lower $F$ transform approximation, i.e. for any $f \in L^{X}$,

$$
d_{k}(f)=\underline{R}_{k}(f) .
$$

Then $d_{k}$ is a Kuratowski fuzzy interior operator in the topology that is determined by fuzzy preorder relation $R_{k}$.
Proof: By Proposition 3.8, operator $d_{k}$ fulfills all properties, listed in Definition 2.8, and the two additional properties, listed in Theorem 2.2. Therefore, $d_{k}$ is a Kuratowski fuzzy interior operator and there exists a fuzzy preorder $R$ on $X$ such that for all $A \in L^{X}, \underline{R}(A)=d_{k}(A)$. It is easy to see that $R_{k}$ can be chosen for $R$.

\section{Inverse $F^{\uparrow}\left(F^{\downarrow}\right)$-transform}

In this section, we show that the inverse $F^{\uparrow}\left(F^{\downarrow}\right)$ transform can be expressed in terms of lower and upper fuzzy approximation operators. We begin with the following concept of an inverse $F^{\uparrow}$ transform.

Definition 4.1 [10] Let $f$ be an L-valued function on $X, A_{1}, \ldots, A_{k}, \ldots$ a fuzzy partition of $X$ and $F^{\uparrow}[f]=\left(F_{1}^{\uparrow}, \ldots, F_{k}^{\uparrow}, \ldots\right)$ the $F^{\uparrow}$-transform of $f$. Then the following function $f_{F}^{\uparrow}: L^{X} \rightarrow L^{X}$, where

$$
f_{F}^{\uparrow}(x)=\bigwedge_{k \geq 1}\left(A_{k}(x) \rightarrow F_{k}^{\uparrow}\right)
$$

is called the inverse $F^{\uparrow}$-transform.

Theorem 4.1 Let $R_{A_{1}, \ldots, A_{k}, \ldots}$ be a representing relation of fuzzy partition $A_{1}, \ldots, A_{k}, \ldots$ of $X$ and $\bar{R}_{A_{1}, \ldots, A_{k}, \ldots}(f)$ be the upper approximation of $f$ in fuzzy approximation space $\left(X, R_{\left.A_{1}, \ldots, A_{k}, \ldots\right)}\right)$. Then the inverse $F^{\uparrow}$-transform of $f$ is equal to

$$
\begin{aligned}
& f_{F}^{\uparrow}(y)= \\
& \bigwedge_{x \in X}\left(R_{A_{1}, \ldots, A_{k}, \ldots}(x, y) \rightarrow \bar{R}_{A_{1}, \ldots, A_{k}, \ldots}(f)(x)\right) .
\end{aligned}
$$

Proof: Let the assumptions be fulfilled. We will prove (13) for arbitrary $y \in X$. Because $A_{1}, \ldots, A_{k}, \ldots$ is a fuzzy partition of $X$, there exists $k \geq 1$, such that $x \in \operatorname{core}\left(A_{k}\right)$. By, (1), $R_{A_{1}, \ldots, A_{k}, \ldots}(x, y)=A_{k}(y)$ and by (5), $\bar{R}_{A_{1}, \ldots, A_{k}, \ldots}(f)(x)=F_{k}^{\uparrow}$. After substitution to the right-hand side of (13) we easily come to the desired equality.

It is easy to see that in the approximation space $\left(X, R_{A_{1}, \ldots, A_{k}, \ldots}^{r e v}\right)$, where $R_{A_{1}, \ldots, A_{k}, \ldots}^{r e v}(x, y)=$ $R_{A_{1}, \ldots, A_{k}, \ldots}(y, x)$, the inverse $F^{\uparrow}$-transform of $f$ is the lower approximation of $\bar{R}_{A_{1}, \ldots, A_{k}, \ldots}(f)$.

In [9], it has been proved that for $f \in L^{X}, f \leq f_{F}^{\uparrow}$. By Proposition 2.3, $f_{F}^{\uparrow} \leq \bar{R}_{A_{1}, \ldots, A_{k}, \ldots}(f)$. Therefore, for every $f \in L^{X}$,

$$
f \leq f_{F}^{\uparrow} \leq \bar{R}_{A_{1}, \ldots, A_{k}, \ldots}(f) .
$$

The similar and dual arguments to that given above, characterizes the inverse $F^{\downarrow}$-transform.

Definition 4.2 [10] Let $f$ be an L-valued function on $X, A_{1}, \ldots, A_{k}, \ldots$ a fuzzy partition of $X$ and 
$F^{\downarrow}[f]=\left(F_{1}^{\downarrow}, \ldots, F_{k}^{\downarrow}, \ldots\right)$ the $F^{\downarrow}$-transform of $f$. Then the following function $f_{F}^{\downarrow}: L^{X} \rightarrow L^{X}$, where

$$
f_{F}^{\downarrow}(x)=\bigvee_{k \geq 1}\left(A_{k}(x) * F_{k}^{\downarrow}\right)
$$

is called the inverse $F^{\downarrow}$-transform.

Theorem 4.2 Let $R_{A_{1}, \ldots, A_{k}, \ldots}$ be a representing relation of fuzzy partition $A_{1}, \ldots, A_{k}, \ldots$ of $X$ and $\underline{R}_{A_{1}, \ldots, A_{k}, \ldots}(f)$ be the lower approximation of $f$ in fuzzy approximation space $\left(X, R_{A_{1}, \ldots, A_{k}, \ldots}\right)$. Then the inverse $F^{\downarrow}$-transform of $f$ is equal to

$$
\begin{aligned}
& f_{F}^{\downarrow}(y)= \\
& \bigvee_{x \in X}\left(R_{A_{1}, \ldots, A_{k}, \ldots}(x, y) * \underline{R}_{A_{1}, \ldots, A_{k}, \ldots}(f)(x)\right) .
\end{aligned}
$$

Proof: Let the assumptions be fulfilled. We will prove (15) for arbitrary $y \in X$. Because $A_{1}, \ldots, A_{k}, \ldots$ is a fuzzy partition of $X$, there exists $k \geq 1$, such that $x \in \operatorname{core}\left(A_{k}\right)$. By, (1), $R_{A_{1}, \ldots, A_{k}, \ldots}(x, y)=A_{k}(y)$ and by $(9)$, $\underline{R}_{A_{1}, \ldots, A_{k}, \ldots}(f)(x)=F_{k}^{\downarrow}$. After substitution to the right-hand side of (15) we easily come to the desired equality.

It is easy to see that in the approximation space $\left(X, R_{A_{1}, \ldots, A_{k}, \ldots}^{r e v}\right)$, where $R_{A_{1}, \ldots, A_{k}, \ldots}^{r e v}(x, y)=$ $R_{A_{1}, \ldots, A_{k}, \ldots}(y, x)$, the inverse $F^{\downarrow}$-transform of $f$ is the upper approximation of $\underline{R}_{A_{1}, \ldots, A_{k}, \ldots}(f)$.

In [9], it has been proved that for $f \in L^{X}, f_{F}^{\downarrow} \leq f$. By Proposition 2.3, $\underline{R}_{A_{1}, \ldots, A_{k}, \ldots}(f) \leq f_{F}^{\downarrow}$. Therefore, for every $f \in L^{X}$,

$$
\underline{R}_{A_{1}, \ldots, A_{k}, \ldots}(f) \leq f_{F}^{\downarrow} \leq f
$$

\section{Concluding Remarks}

In this contribution, we analyzed a mutual relationship among fuzzy rough sets, fuzzy topologies and the $F$-transforms. For this purpose, we generalized the lattice-based $F$-transforms to the case of an arbitrary (not necessary finite) fuzzy partition of a universe. We showed that the direct upper $F^{\uparrow}$ - and lower $F^{\downarrow}$-transforms are respective reductions of upper and lower fuzzy approximation operators. The similar and dual conclusion can be stated for inverse lattice-based $F$-transforms. Moreover, we showed that approximation operators that correspond to particular $F$-transform components are Kuratowski fuzzy interior and closure operators in the induced topologies.

The obtained results nicely connect three different approximation spaces and by this, enable to reduce efforts in proving interrelated statements.

\section{Acknowledgment}

This paper was supported by the European Regional Development Fund in the
IT4Innovations Centre of Excellence project (CZ.1.05/1.1.00/02.0070) and Ministry of Science \& Technology, DST, Govt. of India, under grant No. DST/INSPIRE FELLOWSHIP/2013/502.

\section{References}

[1] D. Boixader, J. Jacas and J. Recasens, Upper and lower approximations of fuzzy sets, International Journal of General Systems, 29:555568, 2000.

[2] R. P. Dilworth and N. Ward, Residuated lattices, Transactions of the American Mathematical Society, 45:335-354, 1939.

[3] D. Dubois and H. Prade, Rough fuzzy sets and fuzzy rough sets, International Journal of General System, 17:191-209, 1990.

[4] J. A. Goguen, L-fuzzy sets, Journal of Mathematical Analysis and Applications, 18:145-174, 1967.

[5] M. Kondo, On the structures of generalized rough sets, Information Sciences, 176:586-600, 2006.

[6] R. Lowen, Fuzzy topological spaces and fuzzy compactness, Journal of Mathematical Analysis and Applications, 56:621-633, 1976.

[7] F. Di Martino, V. Loia, I. Perfilieva and S. Sessa, An image coding/decoding method based on direct and inverse fuzzy transforms, International Journal of Approximate Reasoning, 48:110-131, 2008.

[8] Z. Pawlak, Rough sets, International Journal of Computer and Information Sciences, 11:341356, 1982.

[9] I. Perfilieva and R. Valášek, Fuzzy transforms in removing noise. In B. Reusch, editor, Computational Intelligence, Theory and Applications, pages 221-230, Springer-Verlag, 2005.

[10] I. Perfilieva, Fuzzy transforms: Theory and applications, Fuzzy Sets and Systems, 157:9931023, 2006.

[11] P. Hurtik, and I. Perfilieva, Image compression methodology based on fuzzy transform. In proceedings of the Intern. Conf. on Soft Computing Models in Industrial and Environmental Applications (SoCo2012), Advances in Intelligent and Soft Computing, pages 525-532, Springer-Verlag, 2012.

[12] I. Perfilieva, Fuzzy transforms: A Challenge to Conventional Transforms. In P. W. Hakes, editor, Advances in Image and Electron Physics 147, pages 137-196, Elsevier, San Diego, 2007.

[13] I. Perfilieva, V. Novak and A. Dvorak, Fuzzy transforms in the analysis of data, International Journal of Approximate Reasoning, 48:36-46, 2008.

[14] K. Qin and Z. Pei, On the topological properties of fuzzy rough sets, Fuzzy Sets and Systems, 151:601-613, 2005. 
[15] K. Qin and Z. Pei, Generalized rough sets based on reflexive and transitive relations, Information Science, 178:4138-4141, 2008.

[16] A. M. Radzikowska and E. E. Kerre, Fuzzy rough sets based on residuated lattices. In Transactions on Rough Sets II, Lecture Notes in Computer Science 3135, pages 278-296, Springer-Verlag, 2004.

[17] L. Stefanini, F-transform with parametric generalized fuzzy partitions, Fuzzy Sets and Systems, 180:98-120, 2011.

[18] M. Stepnicka and R. Valasek, Numerical solution of partial differential equations with help of fuzzy transform. In proceedings of the FUZZ-IEEE 2005, Reno, Nevada, Fuzz-IEEE, pages 1104-1109, 2005.

[19] M. Stepnicka and O. Polakovic, A neural network approach to the Fuzzy transform, Fuzzy Sets and Systems, 160:1037-1047, 2009.

[20] Y. H. She and G. J. Wang, An axiomatic approach of fuzzy rough sets based on residuated lattices, Computers and Mathematics with Applications, 58:189-201, 2009.

[21] W. Z. Wu and W.X. Zhang, A study on relationship between fuzzy rough approximation operators and fuzzy topological spaces, Fuzzy Systems and Knowledge Discovery, in: Lecture Notes in Computer Science, 3613 (2005) 167174.

[22] Y. Y. Yao, Constructive and algebraic methods of the theory of rough sets, Information Sciences, 109:21-47, 1998. 